\title{
Medicina en televisión: ¿un problema ético?
}

\author{
Juan Pablo Beca I ${ }^{1}$ y Sofía P. Salas I². \\ Medicine in television. \\ An ethical problem?
}

Television programs where medical procedures are shown with progressive realism generate problems that physicians need to know and analyze. The authors analyze this issue, based on the respect to patient's dignity and the principles of bioethics. Medical programs on TV present specific problems to the different agents involved in them: TV media, physicians, health organization, public and patients or relatives that are exposed. Physicians have the responsibility to educate the society using the most efficient methods of public communication, including television. The problem is not how much can be shown but how to do it, making sure that the dignity of patients, the privacy of their stories and their own and their relative's feelings are always strictly cherished. The respect towards the patient is accomplished through a valid informed consent, the reverence to his face as an expression of his personhood, and the way in which his body is exposed. The authors conclude that TV programs on health and medical subjects are valuable methods to educate society and that physicians, in their function of social educators, should take part in them truly reassuring the respect to patient's dignity and to the bioethical principles of beneficence, autonomy and justice. (Rev Méd Chile 2004; 132: 881-5)

(Key-words: Communications media; Ethics, medical; Mass media; Television)

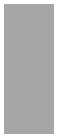

Recibido el 12 de mayo, 2004. Aceptado el 18 de mayo, 2004.

${ }^{1}$ Centro de Bioética, Facultad de Medicina Clínica Alemana - Universidad del Desarrollo.

${ }^{2}$ Departamento de Obstetricia y Ginecología, Escuela de Medicina, Pontificia Universidad Católica de Chile.

$\mathrm{E}^{\mathrm{n}}$ el último tiempo asistimos a la exhibición, cada vez más frecuente, de procedimientos y actos médicos en los medios de comunicación social, muy especialmente en programas de televisión. A las tradicionales series de la década 196069 y las contemporáneas «Emergency Room» y "Chicago Hope», se le han sumado una diversidad de programas de televisión en los cuales partici-

Correspondencia a: Dr. Juan Pablo Beca I. Centro de Bioética, Facultad de Medicina. Clínica Alemana - Universidad del Desarrollo. Vitacura 6082, Vitacura, Santiago Chile. E-mail: jpbeca@udd.cl pan directamente médicos, ya sea como panelistas que responden inquietudes del público o como expertos en programas de difusión científica. Recientemente, y siguiendo la tendencia observada en otros países, han aparecido programas del tipo conocido como «docu-soap» o «reality médiCos», que tienen en común el formato del seguimiento de historias reales. En este género televisivo, los pacientes son escoltados por cámaras desde que ingresan a una consulta o a una sala de urgencia, durante su tratamiento -ya sea médico o quirúrgico- y en su evolución posterior. Es evidente el gran interés del público en recibir 
más información acerca de temas de ciencia y de salud y así, la televisión ha llegado a ser una de las principales fuentes de información pública sobre aspectos biomédicos ${ }^{1}$. Como consecuencia se han generado estrechos vínculos entre el público que desea mayor conocimiento en temas de salud, los médicos, la industria de la salud con sus intereses y las empresas de televisión que buscan captar más televidentes.

Los medios de comunicación, y en este caso la televisión abierta, cumplen un rol social importante al dar información y entretención, a lo cual se agrega una función de educación a la comunidad. Tanto es así, que las principales campañas de prevención en salud, como ha sido por ejemplo la prevención del cólera y del SIDA, utilizan la televisión para promover ciertos cambios en los hábitos de la población. Asimismo, los programas médicos de televisión, si cumplen con fines no sólo de entretención sino también educativos, pueden lograr efectos muy positivo al estimular al público a buscar ayuda médica oportuna para resolver sus problemas de salud y realizar acciones de prevención. Sin embargo, la presentación de algunos programas de televisión, en los que se muestran actos médicos con progresivo realismo, genera dificultades y problemas que son necesarios conocer y analizar. Con este propósito se presenta un análisis de este tipo de programas, tomando como referencia los fundamentos universales de la bioética: respeto a la dignidad del paciente, y los principios bioéticos de beneficencia, autonomía y justicia. Se considerarán los problemas planteados por este tipo de programas, los fines de la medicina, las obligaciones de respeto al paciente y finalmente las recomendaciones internacionales existentes para la filmación de actos médicos.

\section{PROBLEMAS PLANTEADOS POR LOS «REALITY MÉDICOS»}

Los programas de televisión hoy conocidos como género «reality médicos», plantean diversos problemas a los distintos sectores involucrados: los medios de televisión, los médicos, las instituciones de salud, el público $\mathrm{y}$, por último, los pacientes o familiares que se muestran en ellos.

En primer término, los medios de televisión deben velar por la calidad científica de los progra- mas y lograr de esta manera que la información entregada cumpla con un rol educativo y no sólo de entretención. Deben a la vez encontrar las mejores formas de presentación y seleccionar los temas que sean de interés general para el público para asegurar que sean aceptados y alcancen un rating» que les permita obtener su financiamiento. En otras palabras, las empresas de televisión, junto con cumplir con los fines sociales que les son propios, deben cautelar sus propios intereses comenciales. Por su parte, a los médicos, en sus eventuales roles de actores o asesores de prensa, se les plantea el problema de un excesivo protagonismo al convertirse en personajes públicos» sujetos al escrutinio de los televidentes, tanto en sus aspectos profesionales y técnicos, como en otros de su vida privada. Se exponen también a la crítica de sus propios pares, que los pueden descalificar por el excesivo protagonismo que han logrado algunos de los médicos a cargo de estos programas. Un tercer agente son las instituciones de salud que auspician y en cuyas instalaciones se filman estos programas de medicina en televisión. Ellas alcanzan una presencia en la comunidad que difícilmente podrían obtener de otra forma, comunicando a través de la televisión pública sus logros y sus avances tecnológicos y obteniendo así importantes beneficios por la publicidad lograda. El cuarto actor que hemos mencionado es el público que en su hogar puede formarse expectativas imeales con respecto a la solución de sus problemas de salud, lo cual puede generar una dolorosa sensación de frustración y de inequidad en la distribución de los recursos de salud, toda vez que muchos de los avances mostrados difícilmente estarán disponibles para la mayoría de los televidentes. El quinto participante, y sin lugar a dudas el más vulnerable, lo constituye el paciente y su familia, porque son ellos quienes exponen su historia personal, la intimidad de su cuerpo y también de su mente para que a través de ellos se presente un tema o un avance médico. Los motivos por los cuales estos pacientes aceptan esta exposición pueden ser muy diversos, pudiendo ir desde un genuino afán altruista de contribuir con su experiencia al bienestar de otros, hasta un verdadero negocio o transacción comercial, en la cual su participación en el programa está supeditada a ciertas ventajas económicas.

Estamos pues ante intereses y problemas diferentes que afectan a cada uno de estos sectores, 
intereses que a veces llegan a constituir serios conflictos entre ellos. Para llegar a las mejores decisiones es oportuno y necesario que todos, pero muy especialmente los médicos y profesionales de la salud que participan en este tipo de programas, clarifiquen y refuercen sus conceptos sobre los fines últimos de la medicina, las bases de respeto a la dignidad del paciente, y las condiciones que deben tener las filmaciones de actos médicos.

\section{LA MEDICINA Y SUS FINES}

Los objetivos más importantes de la medicina, posiblemente cambiantes en cada época de la historia, han sido replanteados en el proyecto multinacional «The Goals of Medicine» dirigido por Daniel Callahan ${ }^{2}$ y expresados como cuatro fines: 1 ) la prevención de la enfermedad y la promoción de la salud, 2) el alivio del dolor y del sufrimiento causado por enfermedades, 3) el cuidado y curación de quienes padecen enfermedad y el cuidado de los que no pueden ser curados, y 4) la prevención de la muerte prematura y el posibilitar una muerte en paz.

Como estrategia para lograr estos fines, los médicos tienen la responsabilidad de educar a la comunidad, recurriendo para ello a los métodos más eficaces de comunicación social, dentro de los cuales la televisión ocupa indudablemente un papel primordial. Sin embargo, los intereses de la comunidad a la cual se desea educar o entretener no pueden estar por sobre el interés del paciente que participa en estas situaciones como «actor» de estos programas. Por esta razón, es prudente que para cautelar los principios bioéticos universales ya enunciados, no todo lo que suceda en el ámbito del quehacer médico o en la intimidad de la relación médico-paciente sea mostrado por las pantallas de televisión. El tema de fondo no es cuánto ni qué mostrar, sino la forma y condiciones de su presentación. En estas definiciones y decisiones, los médicos deben participar activa y firmemente para priorizar siempre el respeto a la dignidad del paciente.

\section{RESPETO A LA DIGNIDAD DEL PACIENTE Y DE SUS FAMILIARES}

La intimidad de los enfermos, sus historias personales, sus sentimientos y los de su familia, así como su propio cuerpo, merecen el máximo respeto. Los médicos se convierten, de alguna manera, en defensores o guardianes de la dignidad de estos pacientes. Es necesario reflexionar sobre la delgada línea que marca la diferencia entre lo que es conveniente o no exhibir públicamente sobre el quehacer médico. A este respecto hay que destacar dos temas aparentemente distintos, pero que se relacionan directamente con el principio bioético básico de respeto a la dignidad del paciente. Estos se refieren al consentimiento informado y a la imagen del rostro como imagen que singulariza e identifica a la persona.

El concepto del consentimiento informado es más amplio que la simple autorización del paciente o de su familia para participar en un procedimiento médico, y en este caso en un programa de televisión. Para que un consentimiento se pueda considerar válido debe cumplir con varias condiciones, entre otras que no sea obtenido mediante presión o persuasión indebida. Por ejemplo, cuando quien pide esta autorización es el médico tratante, con quien se ha establecido una relación de dependencia y por lo tanto asimétrica, cabe preguntarse si ha existido por parte del paciente y su familia verdadera libertad para decidir. Este tema es especialmente delicado en atenciones de ungencia, porque los pacientes en esa situación pueden no tener la capacidad suficiente para dar su autorización, o bien pueden sentirse presionados a dar su consentimiento al estar sus vidas en manos de quien le están pidiendo su participación ${ }^{3}$. Otra condición en la cual puede limitarse mucho la libertad necesaria para otorgar un consentimiento válido es cuando se ofrecen algunas ventajas para el paciente, tales como rebajas en la cuenta, controles médicos sin costo u otras, condicionadas a su aceptación para ser exhibidos en un programa de televisión.

El otro aspecto en el cual se puede vulnerar el respeto al paciente se refiere al modo de mostrar su cuerpo. La exposición del cuerpo humano en estos programas, lleva a reflexionar sobre cuándo y cuánto se justifica. A este respecto, no parece ser lo mismo la imagen del órgano enfermo, sea éste corazón, hígado, riñón u otro, que la de un rostro sufriente o agonizante. El rostro, a diferencia del órgano, representa a la persona, nos acerca a su humanidad y por eso se debe ser extraordinariamente cuidadoso en determinar cómo se le 
expone, especialmente si el paciente no está consciente de la filmación. Tal como lo señalara Levinas, al Otro se le identifica con el Rostro. \& $E$ l Rostro no se ve, se escucha. El Rostro no es la cara, es la huella del Otro...El Rostro es la palabra del que no posee voz, la palabra del huérfano, de la viuda, del extranjero. El Rostro es significación, y significación sin contexto». En palabras del mismo autor, sel Rostro, es el modo por el cual Otro se presenta y expone su forma, la totalidad de su contenido. El Rostro no es solamente la imagen plástica del Otro, sino más bien todo lo invisible de su vida, la exterioridad de su interioridad, su trascendencia y su libertad $\$$. Por estas consideraciones, la exposición del rostro del paciente, particularmente en situaciones en los cuales se encuentra comprometido de conciencia o próximo a su muerte, debería ser obviada.

De las reflexiones anteriores no se desprende como conclusión una opinión contraria a presentar en televisión programas que exhiban actos 0 procedimientos médicos de manera atractiva $\mathrm{y}$, a veces, con total realismo. Al contrario, los médicos en su rol de educadores sociales deben ofrecer esta información pero deben hacerlo respetando la dignidad del paciente y los principios bioéticos de beneficencia, autonomía y justicia. Para esto se han establecido algunas recomendaciones internacionales que permiten regular la manera de hacerlo.

\section{RECOMENDACIONES AL FILMAR A PACIENTES}

La filmación de pacientes es cada vez más frecuente, sea por necesidades propias del registro clínico de algunos procedimientos, o bien como formas de enseñanza médica y de presentación de casos en actividades académicas. En estas situaciones, generalmente, se tiende a justificar la filmación, así como ocurre con la incorporación de pacientes en proyectos de investigación, por el beneficio social de estas actividades, bajo la estricta condición de que se cuente con su aceptación y que no se afecte su cuidado médico. En el caso de la filmación de pacientes para su presentación en programas públicos, si bien se acepta que existe un beneficio social al educar a los televidentes, algunos consideran que se atenta contra del principio de justicia, puesto que haría que algunos asumen la carga de ser utilizados para enseñar a otros. La dificultad está entonces en lograr un equilibrio entre estos dos bienes aparentemente opuestos: informar al público para lograr un mayor conocimiento médico versus la protección de pacientes individuales.

Se han formulado importantes recomendaciones internacionales sobre el tema, las que conviene conocer y revisar. El Consejo sobre aspectos éticos y judiciales de la Asociación Médica Mundial estableció una serie de recomendaciones al respecto ${ }^{6}$. Los aspectos centrales de esta indicación prohíben expresamente la filmación del paciente sin obtener antes su consentimiento informado, estableciéndose que sólo pacientes capaces de otorgar su consentimiento deben ser filmados. Cuando esto no es posible, sugieren el uso de actuaciones o de recreaciones para evitar así el riesgo de violar la intimidad de un paciente. Agregan que con el objeto de no menoscabar la privacidad del paciente, la filmación debe ocurrir después de otorgado el consentimiento y nunca antes. No aconsejan que un tercero otorgue este consentimiento, salvo en circunstancias excepcionales y determinan que los pacientes tienen el derecho a revocar su consentimiento previo a la salida al aire de un programa en el cual aparecen. Es interesante que esta recomendación de la Asociación Médica de Norteamérica indica que el consentimiento del paciente no debería ser solicitado por el médico tratante sino por un tercero no involucrado.

El Consejo Médico General del Reino Unido establece una detallada guía sobre la filmación con propósitos docentes y luego se refiere explícitamente a la filmación para la exhibición pública de pacientes $^{7}$. Se insiste en este documento que toda vez que un paciente puede ser identificado, su filmación sólo puede hacerse con su consentimiento expreso, asegurándose que haya recibido y comprendido antes claramente los objetivos y alcances de la filmación. Se agrega en esta guía, que los médicos deben estar particularmente atentos a que el bienestar y los intereses del paciente, en especial su privacidad y dignidad, no se comprometan por la filmación o su exhibición. Si esto puede ocurrir, se señala que es responsabilidad del médico detener la filmación o impedir su presentación.

Muchos programas de televisión muestran atenciones de urgencia o de unidades de pacientes críticos, lo cual genera situaciones en las cuales es aún más difícil cautelar el debido 
respeto al paciente, ya sea porque el paciente no se encuentra consciente o porque no está en condiciones de otorgar un consentimiento informado válido. Por esta razón, se ha propuesto filmar primero y luego pedir la autorización, otorgándole al paciente 0 a su familia la posibilidad de retirar este consentimiento. El problema con esta aproximación es que la intimidad del paciente ya ha sido violada, de alguna manera, por el equipo de filmación que no había sido autorizado para estar presente en el acto médico.

\section{CONCLUSIÓN}

Los programas de televisión sobre salud y medicina constituyen un valioso medio de educación

\section{REFERENCIAS}

1. Gerbner G, Gross L, Morgan M, Signoriewi N. Health and medicine on television. N Engl J Med 1981; 305: 901-4.

2. Hanson M, CaLlahan D. The Goals of Medicine: The forgotten issues in health care reform. Georgetown University Press, Washington DC, 1999.

3. Geiderman JM, Larkin GL. Commercial filming of patient care activities in hospitals. JAMA 2002; 288: 373-9. social, favorecen el fomento y protección de la salud y pueden contribuir de manera eficaz a lograr una mejor comprensión de la enfermedad y de sus alternativas de tratamiento. Es responsabilidad de los médicos que participan en estos programas que ellos cumplan con efectivos fines educativos y que no se transformen en un espectáculo televisivo más. Pero por sobre todo, es necesario procurar que en ellos jamás se ofenda, directa 0 indirectamente, la dignidad de algún paciente o de sus familiares. Como médicos, y también como miembros de una institución académica 0 asistencial de salud, debemos reflexionar más sobre los límites que no se pueden transar para lograr que este tipo de programas de televisión tenga éxito y cumpla con propósitos informativos y educativos.

4. Levinas E. Etica e infinito. Gráficas Rógar, SA, Madrid-España, Segunda Edición, página 75; 2000.

5. Levinas E. Totalidad e Infinito. Ensayo sobre la exterioridad, Sígueme, Salamanca; 1977.

6. Council on Ethical and Judicial Affairs, American Medical Association. Filming patients in health care settings. CEJA report 3-1-01, 2001.

7. General MedicAl Council. Making and Using Visual and Audio Recordings of Patients, 2002. www.gmc-uk.org/standards 